\title{
NEW CULLEN PRIMES
}

\author{
WILFRID KELLER
}

\begin{abstract}
Numbers of the forms $C_{n}=n \cdot 2^{n}+1$ and $W_{n}=n \cdot 2^{n}-1$ are both called Cullen numbers. New primes $C_{n}$ are presented for $n=4713,5795$, 6611,18496 . For $W_{n}$, several new primes are listed, the largest one having $n=18885$. Furthermore, all efforts made to factorize numbers $C_{n}$ and $W_{n}$ are described, and the result, the complete factorization for all $n \leq 300$, is given in a Supplement.
\end{abstract}

\section{INTRODUCTION}

In 1905, the Reverend J. Cullen [6] called attention to the numbers $C_{n}=$ $n \cdot 2^{n}+1$, the particular case of $k \cdot 2^{n}+1$ where $k=n$. He observed that $C_{n}$ was composite for all $n$ in the interval $1<n<100$, with the only possible exception of $n=53$. Shortly afterwards, Cunningham [7] gave the prime factor 5591 for $C_{53}$ and restated Cullen's assertion for $1<n \leq 200$, now leaving $n=141$ as the only uncertain case in the considered range. Cunningham pointed out that primes of the form $C_{n}=n \cdot 2^{n}+1$ seemed to be remarkably rare.

Half a century later, in 1957, Robinson [22] showed that $C_{141}$ was in fact a prime. Moreover, he established that this was the only prime $C_{n}$ with $1<n \leq$ 1000. In our numerical investigation we were able to determine four additional primes $C_{n}$. It can now be asserted that $C_{n}$ is prime for $n=1,141,4713$, $5795,6611,18496$, and for no other $n \leq 30000$.

The analogous numbers $W_{n}=n \cdot 2^{n}-1$ have also been investigated. As to their primality, Riesel [21] found in 1968 that $W_{n}$ is prime for $n=2,3,6$, $30,75,81$, but for no other $n \leq 110$. Considerably extending this search, we could add to Riesel's list the primes $W_{n}$ with $n=115,123,249,362,384$, $462,512,751,822,5312,7755,9531,12379,15822,18885$. All other values of $n \leq 20000$ yielded composite numbers.

A comprehensive study of divisibility properties of numbers $C_{n}$ and $W_{n}$ was presented by Cunningham and Woodall (abbreviated C\& W in what follows) in 1917. On account of their classical paper [8], the notations $C_{n}$ (for "Cunningham numbers") and $W_{n}$ (for "Woodall numbers") have been used, while the term Cullen numbers is extended to both these varieties (cf. [13, 24]). $\mathrm{C} \& \mathrm{~W}$ also initiated the project of actually factoring the considered numbers. This work has been extended over the past decades, inasmuch as increasingly

Received by the editor December 3, 1992 and, in revised form, March 28, 1994.

1991 Mathematics Subject Classification. Primary 11A51; Secondary 11B99, 11-04.

Key words and phrases. Cullen numbers, factoring, large primes.

(C)1995 American Mathematical Society 
powerful factorization methods were developed. In a Supplement to this paper the complete factorization of numbers $C_{n}$ and $W_{n}$ is given for all $n \leq 300$.

\section{FACTOR TABLES}

C \& W's report includes the factorization of $C_{n}$ and $W_{n}$ for all $n \leq 32$, except for the single number $W_{30}$, whose primality was not recognized. In the interval $32<n \leq 66$, the character of $W_{n}$ remained doubtful only for $n=39$, 42,51 . The factor table for the original Cullen numbers $C_{n}$ has subsequently been completed at least up to $n \leq 43$ by Beeger [1], to $n \leq 61$ by E. Karst, D. H. Lehmer and J. S. Madachy (as reported in [13]), and to $n \leq 101$ by Steiner [24]. Here, three errata have to be observed: one digit is missing in each of the factorizations given for $n=91,97,101$.

Also from [13] we learn that the factorizations of $W_{n}$ were completed for all $n \leq 49$ by Karst and Madachy, and that Madachy also factored $W_{n}$ for $n=52,55,56,57,59,60$, and 76. The decomposition of $W_{50}$ attributed to Lehmer gives the cofactor $1197765858343217=30503527 \cdot 39266471$ as a prime, and the numbers $W_{123}$ and $W_{249}$ are falsely reported as composites. The factors of $W_{51}$ were later given in [24]. Finally, [13] lists small factors of numbers $C_{n}$ and $W_{n}$ up to $n=300$.

In the Supplement, the complete factorization of both $C_{n}$ and $W_{n}$ is extended to just that limit. The tables were compiled and carefully checked by the author of this paper. Factors for $n \leq 210$ considered difficult to obtain at the time were provided by $\mathrm{G}$. Löh and W. Niebuhr. Thus, Löh completed $C_{n}$ for $102 \leq n \leq 171$ and $W_{n}$ to $n \leq 122$ in 1984, using the methods of [2, $18,20]$, and Niebuhr completed $C_{n}$ and $W_{n}$ for $n \leq 210$ in 1986 with his implementation of the multiple polynomial quadratic sieve (MPQS) based on the description given in [23].

In 1988, $\mathrm{H}$. Suyama supplied the smallest factor of $W_{278}$ and the 16-digit prime factor of $W_{211}$, enabling us to prove primality for the 51-digit cofactor of this number. Suyama used his implementation of the elliptic curve method (ECM), cf. [17]. With only the exception of $W_{211}$, the complete range of $210<$ $n \leq 260$ was done by the author in 1991, using the excellent factorization and prime-testing routines distributed with version 8.15 of Y. Kida's UBASIC, as released in December 1990. For a description of UBASIC, see [19]. The programs ECMX, MPQSX, and MPQSHD, written by Kida, were run on an IBM PS/2-70 386 PC equipped with a mathematical coprocessor. The most demanding application, the decomposition of the 78-digit number $C_{251}$ into its two prime factors with MPQSHD, required 264 hours.

The completion of the final segment $260<n \leq 300$ seemed too hard for UBASIC and was therefore not attempted, leaving unfinished 16 numbers $C_{n}$ and 12 numbers $W_{n}$. They were finally accomplished with the assistance of Niebuhr and R. P. Brent. Niebuhr factorized 21 of the remaining composites, using his own programs for ECM and for MPQS. The latter was run on an IBM ES/9021-440 mainframe. The largest numbers thus treated were the 77-digit cofactors of $C_{261}, W_{264}, W_{267}$, and $W_{289}$, which required about 64 hours of CPU time on the average, and the 79-digit cofactor of $C_{297}$, which took 81 hours.

The other seven composites had $80,81,83$, or 86 digits. All these were 
attacked and finished with Brent's program MVFAC [4], a vectorized implementation of ECM. For details, see [3] and [17]. The program was run by Brent himself on a Fujitsu VP 2200/10 vector processor at the Computer Sciences Laboratory of the Australian National University, and, more intensively, on an SNI S100/10 (similar to a VP 2100/10) recently installed at the Computation Center of the University of Hamburg. Brent obtained the factorization of $W_{277}$ (83-digit cofactor), and the author finished the remaining six numbers. The most notable factor determined with MVFAC was a 39-digit prime factor of $C_{296}$ found after trying only 750 curves. It is also the largest "penultimate" factor contained in Tables I or II of the Supplement. The factorizations for $n \leq 300$ were completed on August 16, 1992 .

Table I of the Supplement includes the factorization of $C_{128}=2^{135}+1$ obtained by Le Lasseur and published by Lucas [16] in 1878 (one digit in the largest factor misprinted), as well as Robinson's prime $C_{141}$. Table II includes the factorization of $W_{64}=2^{70}-1$ already contained in [16], that of $W_{128}=$ $2^{135}-1$ established by P. Poulet in 1946 (see [14]), and the previously known primes. It also includes the factorizations of $W_{100}$ and $W_{256}$, which, according to [13], had been completed by Karst and K. R. Isemonger, respectively. It should be noted that for $n=100,144,196,256$ the factorization of $W_{n}$ was facilitated by an algebraic decomposition, as was the case historically for $C_{128}$ and $W_{128}$.

C \& W listed all 44 values of $n \leq 1000$ for which no factor of $C_{n}$ was known and they showed that in every case the smallest factor $p$ had to be $>1000$. Precisely these $C_{n}$ were tested for primality by Robinson to discover that only $C_{141}$ was prime in that range. Apart from this prime, six more of the $C_{n}$ in question, corresponding to $n=233,245,251,252,285,293$, are in Table I and thus completely factored. In seven cases with $n>300$ the factorization of $C_{n}$ could also be established:

$$
\begin{aligned}
& C_{318}=10939 \cdot 100429275849522701 \cdot \mathrm{p} 78, \\
& C_{436}=1081501579 \cdot 43008589651 \cdot 3717967975567 \cdot \mathrm{p} 102, \\
& C_{579}=4988803100279081295749323 \cdot \mathrm{p} 153, \\
& C_{634}=2459 \cdot 1085629591 \cdot 2756181749 \cdot 7148901709 \cdot \mathrm{p} 162, \\
& C_{753}=164834525239 \cdot \mathrm{p} 219, \\
& C_{921}=2428711 \cdot 2719027 \cdot 4410839 \cdot \mathrm{p} 261, \\
& C_{933}=197724478669 \cdot \mathrm{p} 273 .
\end{aligned}
$$

Here $\mathrm{p} N$ denotes an $N$-digit prime. The seven cofactors, like others to be mentioned below, were proved prime by using the procedure APRT-CL (CohenLenstra version of Adleman-Pomerance-Rumely Test), programmed by K. Akiyama, which is included in Kida's UBASIC. The factorization of $C_{579}$ was obtained through MVFAC.

For 27 of the remaining 30 Cullen numbers $C_{n}$, we give in Table 1 a prime factor. If by trial division a factor $p$ was found below $10^{7}$, the smallest factor of $C_{n}$ is tabulated. If, however, a factor $p>10^{7}$ is given, it was found by one of Pollard's methods or (for $n=581,648,941$ ) by Brent's MVFAC and might not be the least. Thus, only three Cullen numbers $C_{n}$ with $n \leq 1000$ have no known factor, which are for $n=435,453,915$. 
TABLE 1. Prime factors $p$ of Cullen numbers $C_{n}=n \cdot 2^{n}+1$

\begin{tabular}{lrrr}
\hline$n$ & $p$ & $n$ & \multicolumn{1}{c}{$p$} \\
\hline 333 & 2423 & 711 & 367957 \\
402 & 1117 & 713 & 3079 \\
412 & 1091 & 778 & 33667759 \\
473 & 365969 & 816 & 6451 \\
516 & 20641 & 849 & 7103923 \\
532 & 18313 & 869 & 69508729 \\
533 & 95257 & 870 & 39869 \\
580 & 2843 & 899 & 1633716607 \\
581 & 2660379251641 & 900 & 18176209 \\
587 & 60744852593 & 916 & 247451 \\
588 & 203793838081 & 941 & 7717335184972583304615708011 \\
609 & 70038149 & 942 & 2598767 \\
648 & 240383530451966593 & 953 & 381287 \\
693 & 134409623339 & & \\
\hline
\end{tabular}

A prime factor $p<10^{7}$ does exist for all but 41 of the composite numbers $W_{n}$ with $n \leq 1000$. Six of these are completely factored since they have $n \leq 300$. The following six with $n>300$ were factored by finding one factor (in the case of $W_{885}$, MVFAC determined a composite factor):

$$
\begin{aligned}
& W_{369}=7728415141 \cdot \mathrm{p} 104, \\
& W_{463}=141959514756636037 \cdot \mathrm{p} 125, \\
& W_{672}=962794776758617477606791593 \cdot \mathrm{p} 179, \\
& W_{789}=738497627270005859999837 \cdot \mathrm{p} 217, \\
& W_{885}=8353578155864671 \cdot 84769280380290403 \cdot \mathrm{p} 237, \\
& W_{908}=3057961301 \cdot \mathrm{p} 267 .
\end{aligned}
$$

For 23 of the remaining 29, a factor $p>10^{7}$ is presented in Table 2. The factor given for $n=366$ and the smaller factor of $W_{463}$ were kindly supplied

TABLE 2. Prime factors $p$ of Cullen numbers $W_{n}=n \cdot 2^{n}-1$

\begin{tabular}{rrrr}
\hline$n$ & $p$ & $n$ & \multicolumn{1}{c}{$p$} \\
\hline 332 & 12165323 & 675 & 1608969227141 \\
350 & 5169607633 & 722 & 64952161193 \\
366 & 62497690394803 & 723 & 128915821 \\
386 & 119570203 & 765 & 130234223449138177 \\
423 & 3537292571 & 795 & 80958347 \\
522 & 40818521 & 824 & 1757762099 \\
541 & 3241623612714017 & 866 & 10885241 \\
564 & 26768197513 & 906 & 16556069 \\
570 & 48623921 & 931 & 15127751 \\
621 & 16127089673 & 932 & 114082154860229 \\
663 & 60166683064673819 & 943 & 405108238890652513 \\
669 & 73399869877 & & \\
\hline
\end{tabular}


by H. Suyama, who found them in 1988. Those for $n=541,663,765$, 932,943 are due to MVFAC, like the above factors of $W_{672}$ and $W_{789}$. The numbers $W_{n}$ without a known factor now occur for $n=349,375,668,715$, $951,963$.

There is presently a project underway attempting complete factorizations for the whole range $300<n \leq 1000$. As of the end of February 1994, 147 numbers $C_{n}$ and 142 numbers $W_{n}$ had virtually been finished. A machine readable list with all known factors is available from the author, who would welcome any new factors that readers may wish to contribute.

\section{DiVISIBILITY PROPERTIES}

In pursuance of the original observations made by Cullen and by Cunningham, a glance at the factor table for $C_{n}$ suggests that a prime $p$ divides both the numbers $C_{p-1}$ and $C_{p-2}$. A more general statement is in fact true. For a given odd prime $p$ let $n_{k}=\left(2^{k}-k\right)(p-1)-k, k \geq 0$. This includes $n_{0}=p-1$ and $n_{1}=p-2$. The statement is that $p$ divides $C_{n_{k}}$ for all $k \geq 0$, and it is verified easily. Since $n_{k} \equiv-2^{k}(\bmod p)$, we have $n_{k} \cdot 2^{n_{k}} \equiv-2^{k+n_{k}} \equiv-2^{\left(2^{k}-k\right)(p-1)}$ $(\bmod p)$, which by Fermat's theorem is congruent to -1 modulo $p$. Therefore $C_{n_{k}} \equiv 0(\bmod p)$. Obviously, $n_{k}$ cannot be a multiple of $p$.

To describe the totality of numbers $C_{n}$ that are divisible by a given prime $p$, two remarks are in order. First, the numbers $C_{n} \bmod p$ are periodic with period $p h_{p}$, where $h_{p}=\exp _{p}(2)$ is the smallest positive integer $h$ such that $2^{h} \equiv 1(\bmod p)$. As a consequence, if $p$ divides $C_{n}$ for some $n$, then $p$ also divides $C_{n+p h_{p}}$. Secondly, from the definition of $n_{k}$ it may be deduced that there are exactly $h_{p}$ numbers $n_{k}$ which are incongruent modulo $p h_{p}$, and no other $n$ with $0<n<p h_{p}$ gives a $C_{n}$ with a prime factor $p$ (cf. [8]). So, the totality of all $n$ such that $C_{n}$ is divisible by $p$ is obtained as follows. Determine $n_{k}^{\prime}=n_{k} \bmod p h_{p}$ for $k=0,1, \ldots, h_{p}-1,0<n_{k}^{\prime}<p h_{p}$. Then, for every $k$, include $n=n_{k}^{\prime}+r p h_{p}$ for all $r \geq 0$.

In particular, the prime $p=3$ divides all $\bar{C}_{n}$ with $n \equiv 1,2(\bmod 6)$. As a further example, for $p=11, h_{p}=10$, we get $n_{k}^{\prime}=10,9,18,47,6,45$, $24,103,52,71$, and all integers $n$ congruent modulo 110 to any one of these.

A similar description can be given for the set of subscripts $n$ for which $W_{n}$ is divisible by $p$. Let $n_{k}=-\left(2^{k}+k\right)(p-1)-k$ (these are negative integers) for $k \geq 0$, determine $n_{k}^{\prime}=n_{k} \bmod p h_{p}$ for $k=0,1, \ldots, h_{p}-1,0<n_{k}^{\prime}<p h_{p}$, and, for every $k$, include $n=n_{k}^{\prime}+r p h_{p}$ for all $r \geq 0$. In this case, $p=3$ divides all $W_{n}$ with $n \equiv 4,5(\bmod 6)$, and $p=11, h_{p}=10$ give $n_{k}^{\prime}=100$, $79,48,107,16,65,64,73,102,61$, and all $n$ congruent modulo 110 to any one of these.

Note that for $n=65,64$ we have two consecutive numbers $W_{n}$, and actually an infinity of such pairs, which are both divisible by the same prime $p=11$. For numbers $C_{n}$ this situation occurs for every prime $p$, as we have seen. For numbers $W_{n}$, however, this is restricted to the case that $h_{p}$ is an even number. The minimality of $h_{p}$ then implies that $2^{h_{p} / 2} \equiv-1(\bmod p)$ and thus also $2^{p h_{p} / 2} \equiv-1(\bmod p)$. It is now easily seen that $W_{n} \equiv 0(\bmod p)$ for $n=p h_{p} / 2+p-1$ and for $n=p h_{p} / 2+p-2$.

Other divisibility rules are also contained in the given general description. 
For instance, $p$ divides $C_{(p+1) / 2}$ and $W_{(3 p-1) / 2}$, or it divides $C_{(3 p-1) / 2}$ and $W_{(p+1) / 2}$, according as the Jacobi symbol $(2 / p)$ is -1 or +1 . Again, this can be verified immediately. For more details, see [7] and [8].

In spite of the apparent similarity in the description of small factors for both sequences $\left\{C_{n}\right\}$ and $\left\{W_{n}\right\}$, considerably more composites are obtained for the original Cullen numbers $C_{n}$. This is primarily due to the fact that every prime $p>3$ immediately gives four consecutive composite numbers $C_{n}$. In addition to $C_{p-1}$ and $C_{p-2}$, always two neighboring numbers of the sequence are divisible by 3 . If $p$ is of the form $p=6 k+1$, then 3 divides $C_{p}$ and $C_{p+1}$, and in the case of $p=6 k-1$, the prime 3 divides $C_{p-3}$ and $C_{p-4}$. In the special situation of a twin prime pair $p, q=6 k \pm 1$, together a string of eight consecutive composites $C_{n}$ occurs.

To give an indication of how the notable difference in the number of primes of the forms $C_{n}$ and $W_{n}$ comes about, let us first consider all values of $n \leq 110$ such that $C_{n}$ has no prime factor $p \leq n+2$. Here we get $n=33,53,75$, where $n=75$ can be eliminated through the prime factor $p=2 n-1=149$. In the case of $W_{n}$, however, 32 values of $n$ remain in the first step, of which eight are eliminated by a factor $p=2 n-1$. The remainder of 24 includes, of course, the six values of $n \leq 110$ giving rise to the primes $W_{n}$ determined by Riesel.

\section{CUllen PRimes}

In our search for new primes, we tested $C_{n}$ for $n \leq 20000$ in 1984 and continued (without success) to $n \leq 30000$ in 1987 . Similarly, $W_{n}$ was tested up to $n \leq 15000$ in 1984, and to $n \leq 20000$ in 1987. In this case two more primes were found for $n=15822$ and $n=18885$. All other new Cullen primes were thus discovered in 1984 . The methods used for proving primality are found in [5]. For $1000<n \leq 20000$, a total of 632 numbers $C_{n}$ and 1203 numbers $W_{n}$ had actually to be tested after the sieving procedure. In addition, 321 numbers $C_{n}$ with $20000<n \leq 30000$ remained without known factor. We also considered the possibility of $n \cdot 2^{n} \pm 1$ forming a pair of twin primes. Such "Cullen twins" may indeed exist for some large $n$. But we showed (by testing individual numbers $C_{n}$ ) that this $n$ must exceed $n=41528$.

The prime $C_{18496}=17^{2} \cdot 2^{18502}+1=\left(17 \cdot 2^{9251}\right)^{2}+1$ might be of some interest in relation to Conjecture E of Hardy and Littlewood [10], which states that an infinity of primes $N^{2}+1$ should exist (actually, the conjecture was given in a quantitative asymptotic form). At the time of its discovery, our prime seemed to be the largest one known of that expression. But now we know five larger ones, discovered by $\mathrm{H}$. Dubner (unpublished), which are of the form $b^{2^{m}}+1$, with $b$ even. The largest of these primes is $200944^{2^{11}}+1$, which has 10861 digits and was found in September, 1992. Incidentally, Dubner [9] has also considered a generalization of Cullen numbers given by $n \cdot b^{n}+1$ for $b>2$.

Regarding the primes $W_{n}$ listed for $n>110$, it appeared that four of them had already been known for some time. As a matter of fact, Williams and Zarnke [25] had found $W_{115}$ to be prime, Jönsson [12] had found $181 \cdot 2^{363}-1=$ $W_{362}$, Riesel [21] had given $3 \cdot 2^{391}-1=W_{384}$, and $W_{512}$ turned out to be the Mersenne prime $M_{521}=2^{521}-1$ discovered by Robinson (see [15]).

A first result concerning the distribution at large of Cullen primes $C_{n}$ was obtained by Hooley [11]. By applying sieve methods, he showed that the natural 
density of positive integers $n \leq x$ for which $C_{n}$ is a prime is of the order $o(x)$ for $x \rightarrow \infty$. In that sense, almost all Cullen numbers are composite. Suyama (communicated personally) has reworked Hooleys proof to show that it is applicable to any sequence of numbers $n \cdot 2^{n+a}+b$, where $a, b$ are arbitrarily fixed integers. In particular, Hooley's result also follows for numbers $W_{n}$.

In the context of a search for prime factors of Fermat numbers our particular interest in Cullen numbers $C_{n}$ emanated from the remark of $\mathrm{C} \& \mathrm{~W}$, supported by an explicit argument, that primes of the form $C_{n}$ seemed likely as possible divisors of Fermat numbers. However, the four new primes $C_{n}$ proved not to divide any of these.

Instead, there is some evidence for the likelihood that a prime $W_{n}$ divides a Mersenne number $M_{p}$. We noticed that $W_{2}=M_{3}$ and $W_{512}=M_{521}$, and that $W_{3}$ divides $M_{11}, W_{6}$ divides $M_{191}$, and $W_{123}$ divides $M_{p}$ for $p=$ $123 \cdot 2^{122}-1$, a 39-digit prime. In all three cases where $W_{n}$ properly divides $M_{p}$, we have $p=\left(W_{n}-1\right) / 2=n \cdot 2^{n-1}-1$, that is, $p$ and the divisor $W_{n}=2 p+1$ are both primes.

To establish in general whether $W_{n}$ divides any $M_{p}$ or not, the complete factorization of $\left(W_{n}-1\right) / 2$, if available, can be used. In fact, a divisor $W_{n}$ of $M_{p}$ must also be of the form $2 k p+1$. Equivalently, $\left(W_{n}-1\right) / 2=k p$, hence the particular subscript $p$ should divide $\left(W_{n}-1\right) / 2$. So, for all different prime factors $p$ of this number, one would have to check if $M_{p} \bmod W_{n}=0$. But in practice a much easier test suffices, as Suyama has indicated in a personal communication. To see whether a prime $q$ divides some Mersenne number or not, only an "evenly factored portion" of $q-1$ and its cofactor need to be used.

Let $q$ be a prime and $q-1=2^{\alpha_{0}} \cdot p_{1}^{\alpha_{1}} \cdot p_{2}^{\alpha_{2}} \cdots \cdot p_{r}^{\alpha_{r}} \cdot C$, where $p_{j}$ are odd primes and $C$ is composite. If $2^{p_{j}} \not \equiv 1(\bmod q)$ for $1 \leq j \leq r$ and $2^{C} \not \equiv 1$ $(\bmod q)$, then $q$ cannot be a factor of any Mersenne number. In the alternative case that $2^{C} \equiv 1(\bmod q), q$ may be a factor of $M_{p}$, where $p$ is some prime factor of $C$. Indeed, if for an unknown factor $p$ of $C$ we had $q \mid M_{p}$, that is $2^{p} \equiv 1(\bmod q)$, then necessarily $2^{C} \equiv 1(\bmod q)$.

For all primes $q=W_{n}$ with $30 \leq n \leq 822$ (except for the otherwise settled $n=123$ ) this test was conclusive by using only one odd factor $p_{1}$ of $W_{n}-1$. In most cases, a very small $p_{1}$ was readily found, while for $n=249$ and $n=384$ the factors $p_{1}=708211533214392631$ resp. $p_{1}=12694590781$ determined by Suyama had to be used. It has also been checked that the Cullen number $W_{5312}$ does not divide any Mersenne number, by calculating $2^{C} \bmod W_{5312}$ for $C=\left(W_{5312}-1\right) /\left(2 \cdot 3 \cdot 5^{2}\right)$. But here we had the interesting case that, nevertheless, $2^{C^{\prime}} \equiv 1\left(\bmod W_{5312}\right)$ for $C^{\prime}=\left(W_{5312}-1\right) /(2 \cdot 3)$. For $W_{7755}$, the test could not be carried out, because no factor of the composite number $\left(W_{7755}-1\right) / 2$ was found. Recently, Dubner has kindly tested the numbers $W_{n}$ for $n=9531$, $12379,15822,18885$, without detecting a divisibility. In the case of $n=$ 15822 he obtained $2^{C} \equiv 1\left(\bmod W_{15822}\right)$, where $C=\left(W_{15822}-1\right) /(2 \cdot 7)$ and no further factor of this number was known.

Of course, $W_{n}$ may also be identical to a Mersenne number without being a prime. Generally, $W_{n}$ is a Mersenne number whenever $n=2^{m}$ and $m+2^{m}$ is prime. This is the case for $m=1: W_{2}=M_{3}=7 ; m=3: W_{8}=M_{11}=23.89$; $m=5: W_{32}=M_{37}=223 \cdot 616318177 ; m=9: W_{512}=M_{521} ;$ and also for $m=15,39,75,81,89,317,701,735$ and no other $m \leq 1310$. 


\section{BIOGRAPHICAL NOTE}

When we searched the literature for references to Cullen numbers, we were intrigued by the fact that almost nothing could be found about Cullen as a person. Even mathematical or encyclopaedic dictionaries usually giving such information failed to disclose Cullen's complete first name or to mention the year of his birth or his death. Only the abbreviation "S. J." placed after his name in a footnote to [8] suggested that he was a Jesuit priest. That hint finally enabled us to locate the most appropriate and authoritative source for this matter, namely, the Department of Historiography and Archives of the English Province of the Society of Jesus. The archivist of that institution, Father T. G. Holt, was kind enough to provide the following biographical data, which we are pleased to present to the reader in unabridged form.

Father James Cullen, S. J., was born on April $19^{\text {th }} 1867$ at Drogheda in Ireland. At first he was educated privately, then by the Christian Brothers. Next he went to Trinity College, Dublin, to study pure and applied Mathematics. Afterwards he was in business for a while, but after a short period at an apostolic school in Ireland to learn Latin he entered the noviceship of the English Jesuits at Manresa House, Roehampton, London, as he had decided to become a priest. From 1892 till 1895 he was at Manresa in the noviceship and then for a year's study. From 1895 till 1897 he studied philosophy at the Jesuit house for philosophical studies at St Mary's Hall, Stonyhurst in Lancashire. From 1897 till 1901 he studied theology at the theologate of the English Jesuits at St Beuno's College in North Wales. He was ordained priest at St Beuno's College on July $31^{\text {st }} 1901$. In 1902 and 1903 he taught Mathematics to young Jesuits who had finished their noviceship at Manresa House. In 1905 he taught Mathematics at the Jesuit boarding school Mount St Mary's College in Derbyshire. It is said that he found difficulty in teaching. In 1906 he was sent to Stonyhurst College, also a boarding school, as accountant and later manager of the College farm and estate. Meanwhile he kept in touch with leading mathematicians and contributed articles to Nature, the Mathematical Gazette and the Messenger of Mathematics. In 1921 he left Stonyhurst and was appointed to supervise accounts of other English Jesuit houses. He died on December $7^{\text {th }} 1933$.

\section{ACKNOWLEDGMENTS}

The author thanks Hiromi Suyama for his remarks and suggestions indicated in the text, as well as for the provision of some prime factors, and to Günter Löh for the factorizations mentioned in $\S 2$. Thanks are also due to Wolfgang Niebuhr for his willingness to produce difficult factorizations whenever asked to do so. He has been appropriately included as a coauthor of the Supplement in acknowledgment of the substantial contributions he has made to it over the years. Special thanks are extended to Richard Brent for making available his program MVFAC and thus allowing us to obtain the last factorizations needed to complete the Supplement. 
Finally, the author is greatly indebted to Father Bruno Brinkman of Heyihrop College, University of London, for helping to establish contact with Father Geoffrey Holt, who compiled and wrote the enlightening biographical note of $\S 5$.

\section{BIBLIOGRAPHY}

1. N. G. W. H. Beeger, Cullen numbers, MTAC 8 (1954), 188.

2. R. P. Brent, An improved Monte Carlo factorization algorithm, BIT 20 (1980), 176-184.

3. __ Some integer factorization algorithms using elliptic curves, Austral. Comput. Sci. Comm. 8 (1986), 149-163.

4. , MVFAC: A vectorized Fortran implementation of the elliptic curve method, Comput. Sci. Lab., Austral. Nat. Univ., 1991.

5. J. Brillhart, D. H. Lehmer, J. L. Selfridge, B. Tuckerman, and S. S. Wagstaff, Jr., Factorizations of $b^{n} \pm 1, b=2,3,5,6,7,10,11,12$ up to high powers, 2nd ed., Contemp. Math., vol. 22, Amer. Math. Soc., Providence, RI, 1988.

6. J. Cullen, Question 15897, Educ. Times, Dec. 1905, 534.

7. A. Cunningham, Solution of Question 15897, Math. Quest. Educ. Times 10 (1906), 44-47.

8. A. Cunningham and H. J. Woodall, Factorisation of $Q=\left(2^{q} \mp q\right)$ and $\left(q .2^{q} \mp 1\right)$, Messenger Math. 47 (1917), 1-38.

9. H. Dubner, Generalized Cullen numbers, J. Recreational Math. 21 (1989), 190-194.

10. G. H. Hardy and J. E. Littlewood, Some problems of 'Partitio numerorum'; III: On the expression of a number as sum of primes, Acta Math. 44 (1923), 1-70.

11. C. Hooley, Applications of sieve methods to the theory of numbers, Cambridge Univ. Press, Cambridge, 1976.

12. I. Jönsson, On certain primes of Mersenne-type, BIT 12 (1972), 117-118.

13. E. Karst, Prime factors of Cullen numbers $n \star 2^{n} \pm 1$, Number Theory Tables, compiled by A. Brousseau, Fibonacci Assoc., San Jose, Calif., 1973, pp. 153-163.

14. D. H. Lehmer, On the factors of $2^{n} \pm 1$, Bull. Amer. Math. Soc. 53 (1947), 164-167.

15. __ Recent discoveries of large primes, MTAC 6 (1952), 61.

16. E. Lucas, Sur la série récurrente de Fermat, Bull. Bibl. Storia Sc. Mat. Fis. 11 (1878), 783-798.

17. P. L. Montgomery, Speeding the Pollard and elliptic curve methods of factorization, Math Comp. 48 (1987), 243-264.

18. M. A. Morrison and J. Brillhart, $A$ method of factoring and the factorization of $F_{7}$, Math. Comp. 29 (1975), 183-205.

19. W. D. Neumann, UBASIC: a Public-Domain BASIC for Mathematics, Notices Amer. Math. Soc. 36 (1989), 557-559; UBASIC Update, ibid. 38 (1991), 196-197.

20. J. M. Pollard, Theorems on factorization and primality testing, Proc. Cambridge Philos. Soc. 76 (1974), 521-528.

21. H. Riesel, Lucasian criteria for the primality of $N=h \cdot 2^{n}-1$, Math. Comp. 23 (1969), 869-875.

22. R. M. Robinson, A report on primes of the form $k \cdot 2^{n}+1$ and on factors of Fermat numbers, Proc. Amer. Math. Soc. 9 (1958), 673-681.

23. R. D. Silverman, The multiple polynomial quadratic sieve, Math. Comp. 48 (1987), 329-339.

24. R. P. Steiner, On Cullen numbers, BIT 19 (1979), 276-277.

25. H. C. Williams and C. R. Zarnke, $A$ report on prime numbers of the forms $M=(6 a+1) 2^{2 m-1}$ -1 and $M^{\prime}=(6 a-1) 2^{2 m}-1$, Math. Comp. 22 (1968), 420-422.

Regionales Rechenzentrum der Universität Hamburg, 20146 Hamburg, Federal RePUBLIC OF GERMANY

E-mail address: keller@rrz.uni-hamburg.de 
MATHEMATICS OF COMPUTATION

VOLUME 64, NUMBER 212

OCTOBER 1995, PAGES S39-S46

Supplement to

NEW CULLEN PRIMES

WILFRID KELLER AND WOLFGANG NIEBUHR

TABLE I

Factorization of Cullen numbers $C_{n}=n \cdot 2^{n}+1$

n $\quad C_{n}=n \cdot 2^{n}+1$

13

$2 \quad 3^{2}$

$3 \quad 5^{2}$

$45 \cdot 13$

$5 \quad 7 \cdot 23$

$6 \quad 5 \cdot 7 \cdot 11$

$7 \quad 3 \cdot 13 \cdot 23$

$\begin{array}{ll}8 & 3 \cdot 683\end{array}$

$9 \quad 11.419$

$10 \quad 7^{2} \cdot 11 \cdot 19$

$11 \quad 13 \cdot 1733$

$12 \quad 13 \cdot 19 \cdot 199$

$13 \quad 3^{2} \cdot 11833$

$14 \quad 3 \cdot 157 \cdot 487$

$15 \quad 17 \cdot 29 \cdot 997$

$16 \quad 17 \cdot 61681$

$17 \quad 5^{2} \cdot 19 \cdot 4691$

$18 \quad 11 \cdot 19 \cdot 107 \cdot 211$

$19 \quad 3 \cdot 17 \cdot 37 \cdot 5279$

$20 \quad 3^{3} \cdot 103 \cdot 7541$

$21 \quad 23 \cdot 1914791$

$22 \quad 13 \cdot 23 \cdot 43 \cdot 7177$

$23 \quad 5 \cdot 151 \cdot 421 \cdot 607$

$24 \quad 5 \cdot 11 \cdot 1399 \cdot 5233$

$25 \quad 3 \cdot 17 \cdot 2459 \cdot 6689$

$26 \quad 3 \cdot 5 \cdot 7^{2} \cdot 2373919$

$27 \quad 7 \cdot 29 \cdot 53 \cdot 336823$

$28 \quad 29^{2} \cdot 8937209$

$29 \quad 31 \cdot 83 \cdot 6051013$

$30 \quad 17^{2} \cdot 31 \cdot 59 \cdot 149 \cdot 409$

$31 \quad 3^{2} \cdot 7 \cdot 61 \cdot 1483 \cdot 11681$

$32 \quad 3 \cdot 1777 \cdot 25781083$

$33 \quad 47 \cdot 6031230671$

$34 \quad 19 \cdot 23 \cdot 67 \cdot 1061 \cdot 18803$

$35 \quad 37 \cdot 32502455213$

$36 \quad 37^{2} \cdot 1933 \cdot 934861$

$37 \quad 3 \cdot 5 \cdot 339016085231$

$38 \quad 3^{2} \cdot 20879 \cdot 55586743$

$39 \quad 41 \cdot 3433 \cdot 152326961$

$40 \quad 41 \cdot 131611 \cdot 8150491$ 


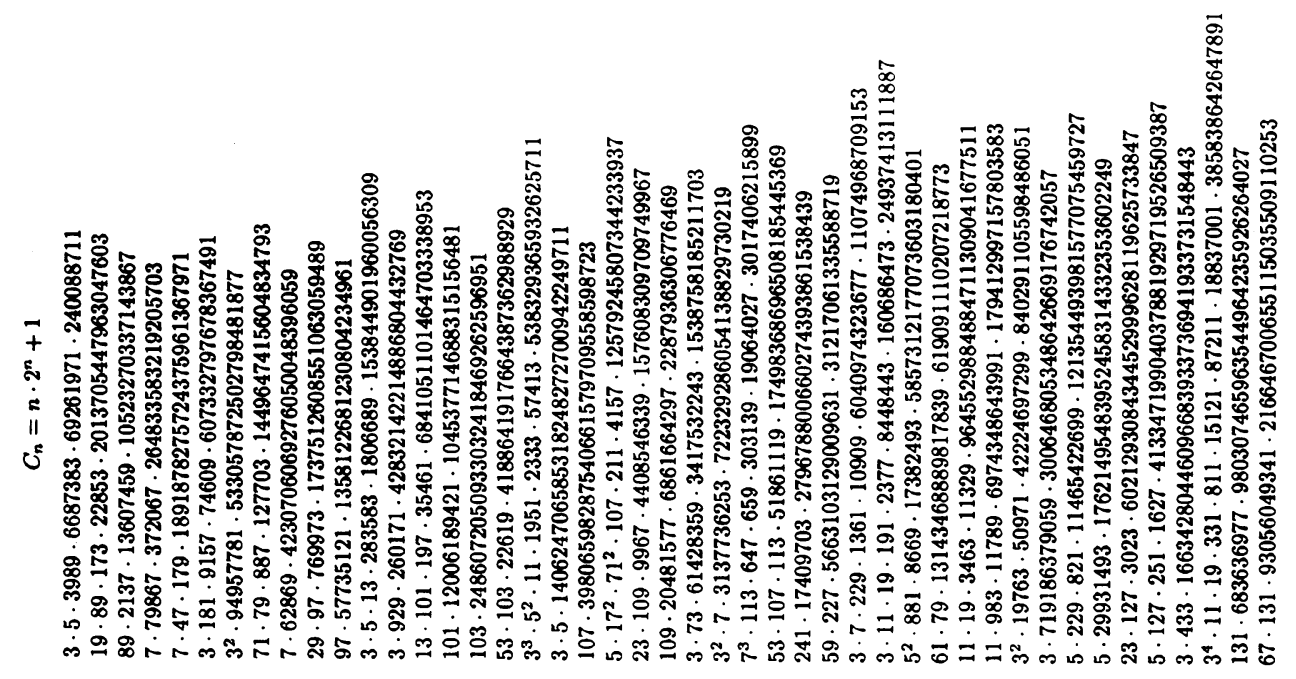

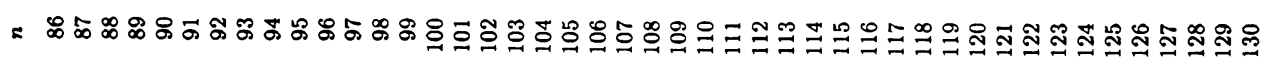

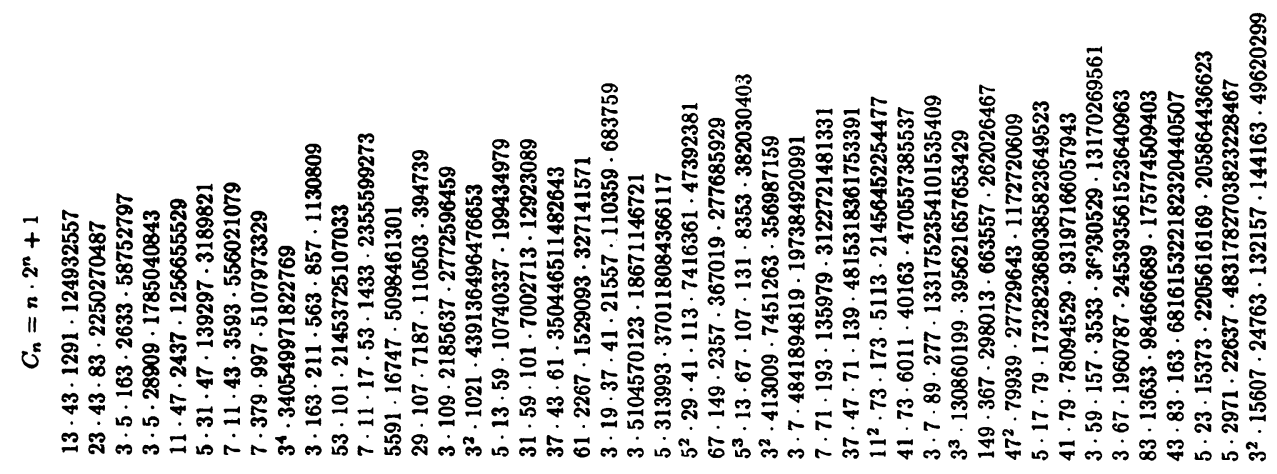

こ テ พ 


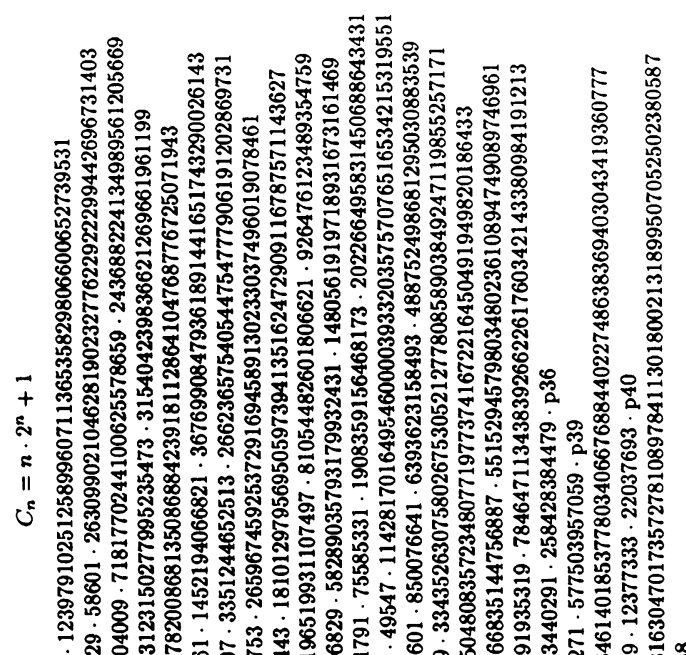

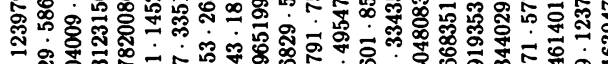

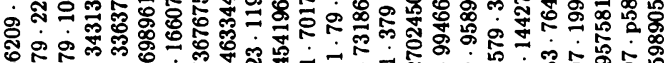

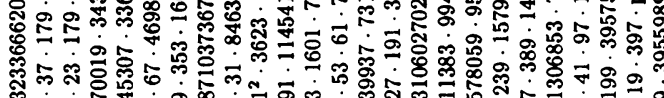

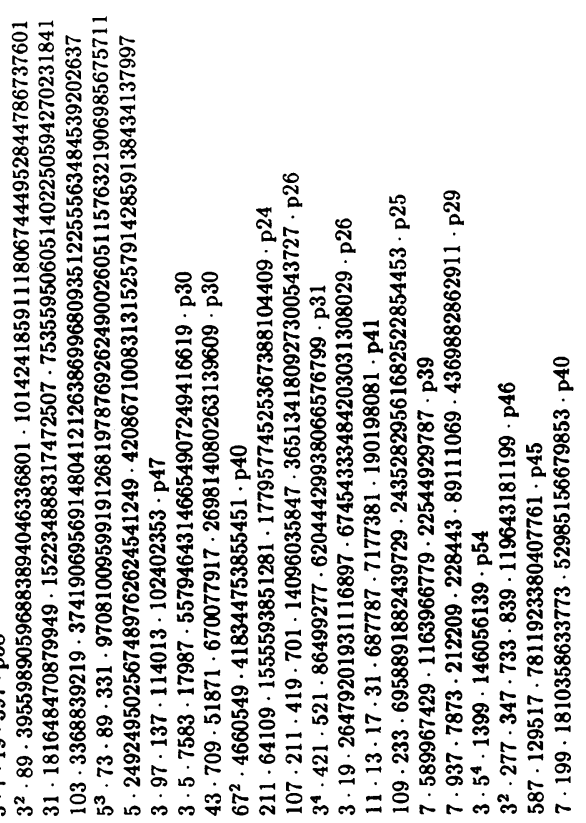

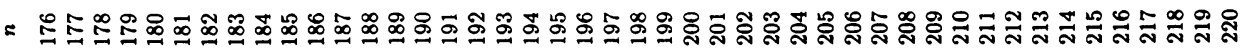

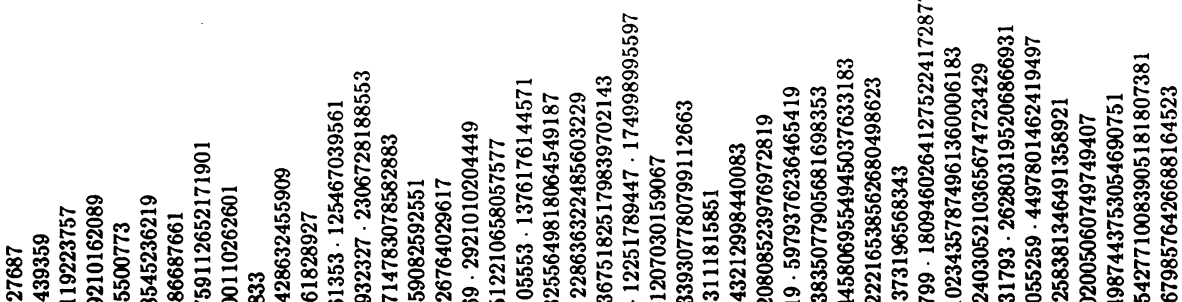

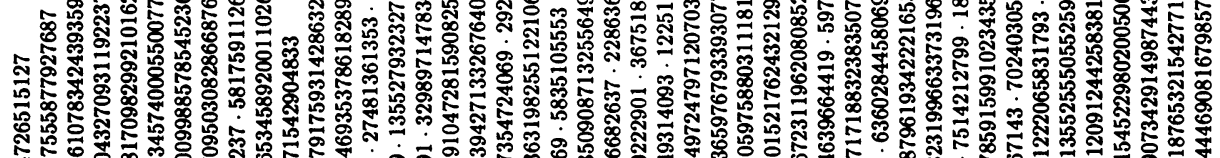
† 赵点 +

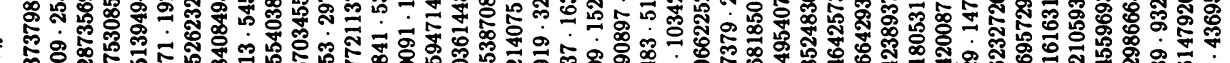

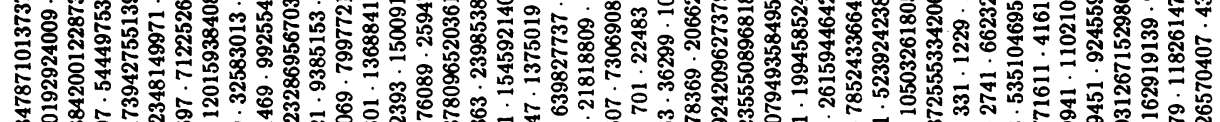

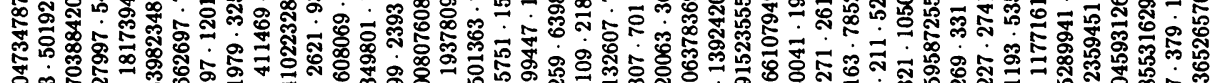

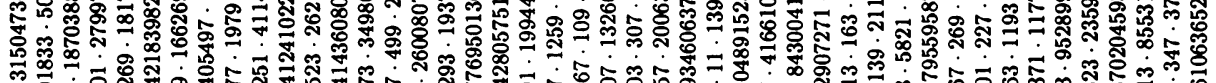

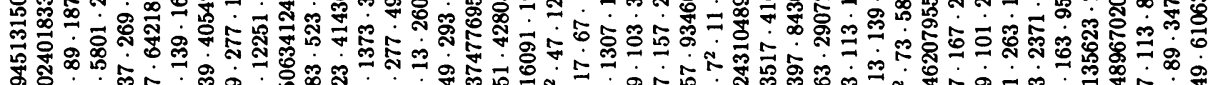

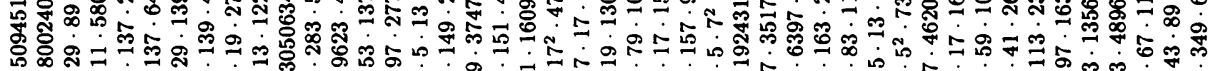

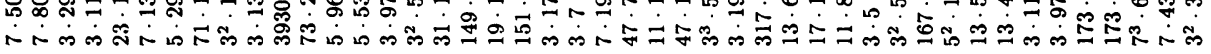

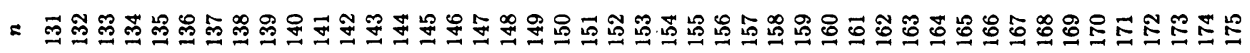




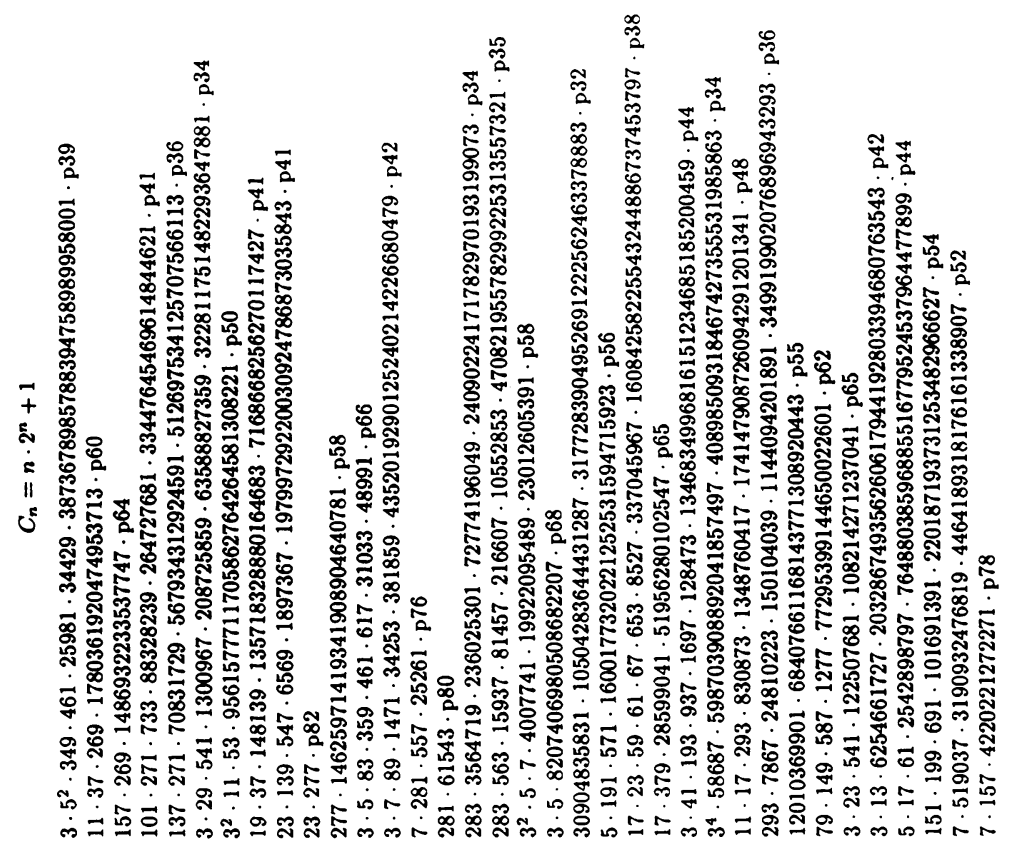

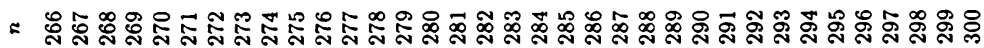

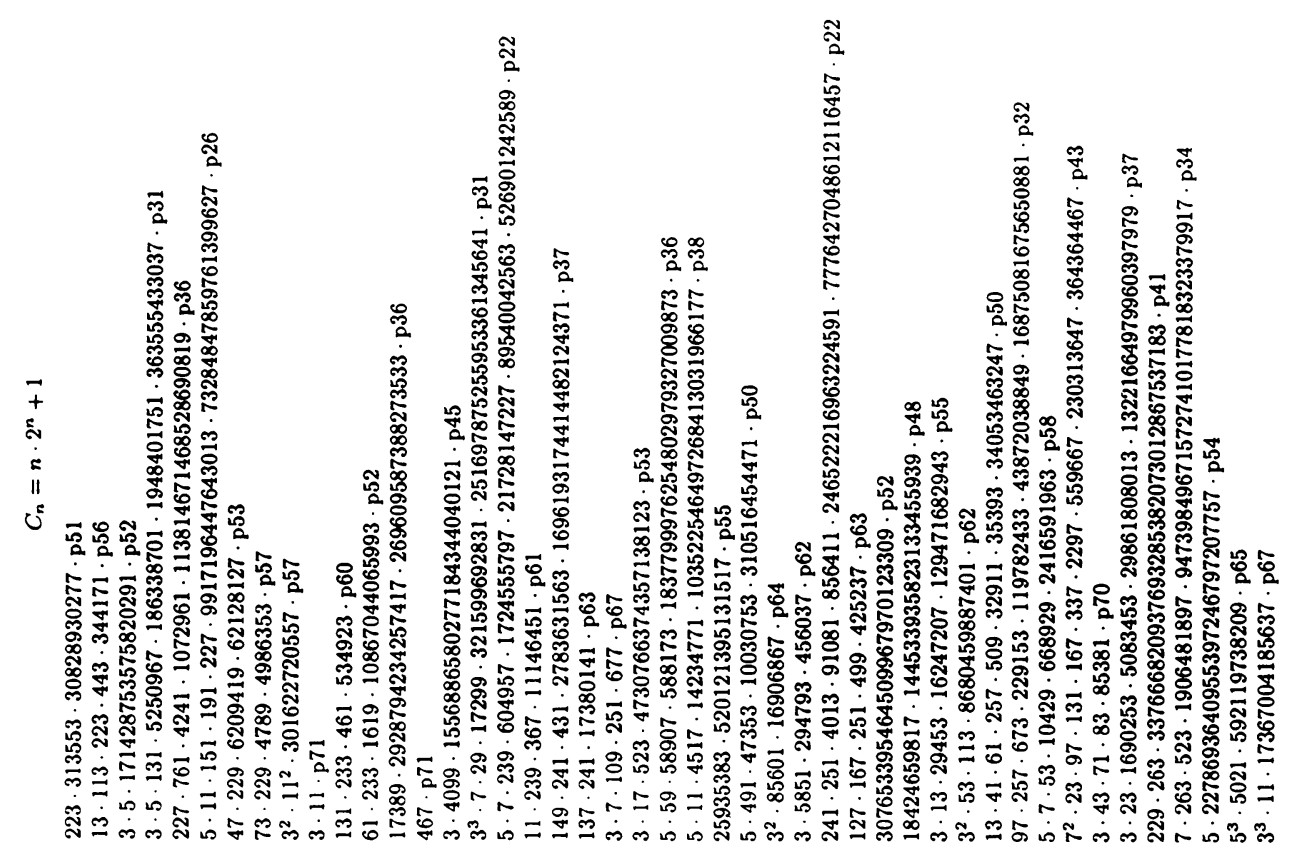

— 

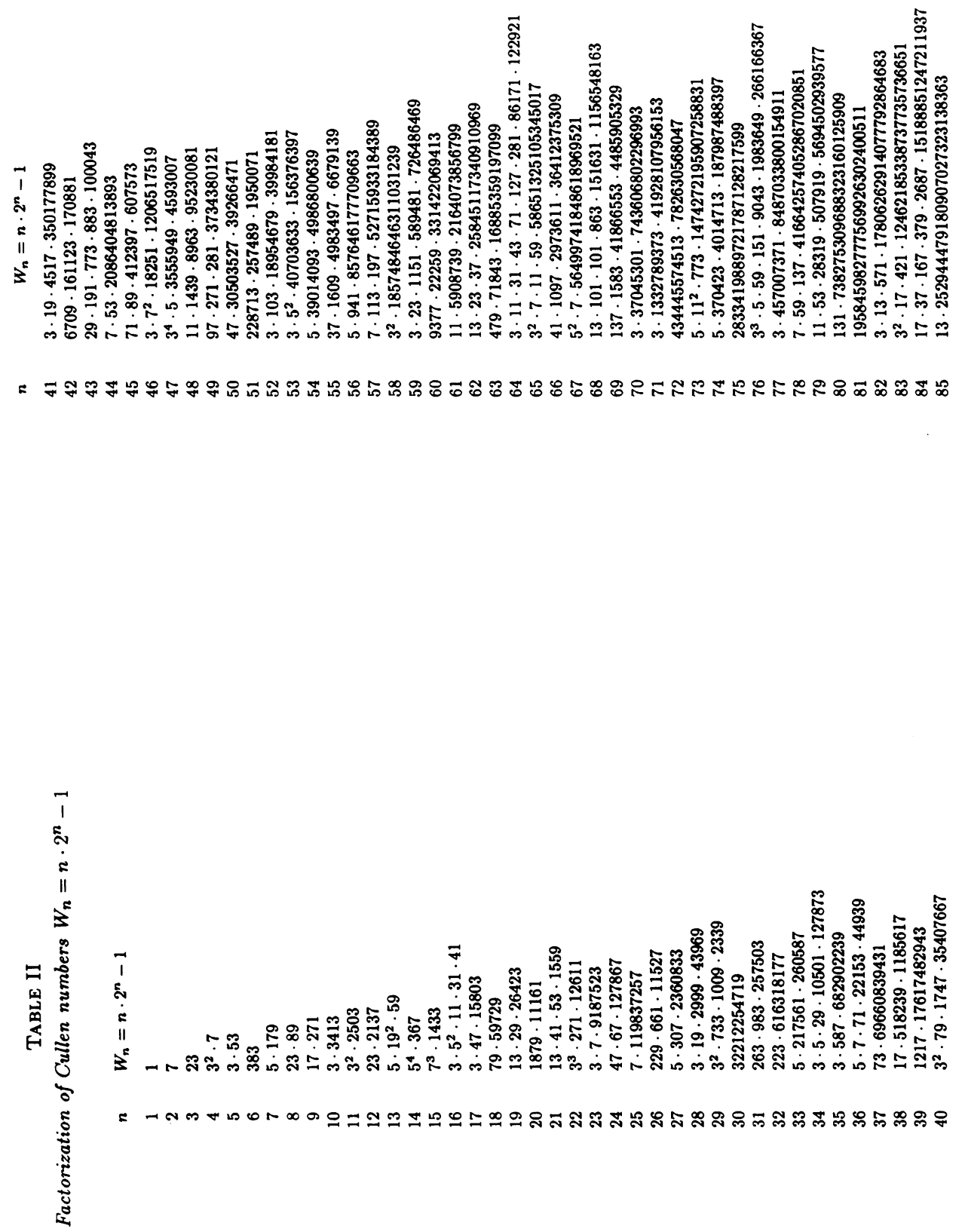
।

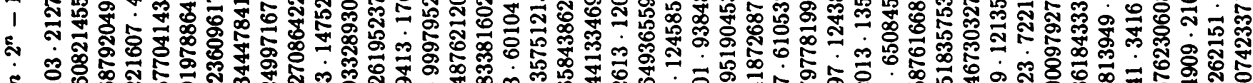

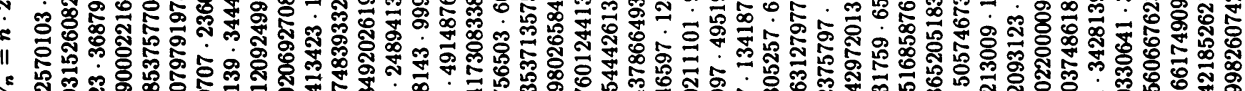
"

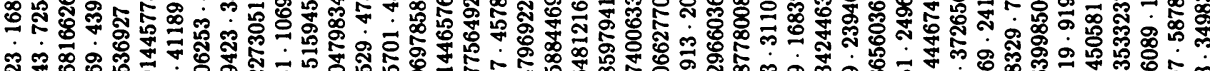

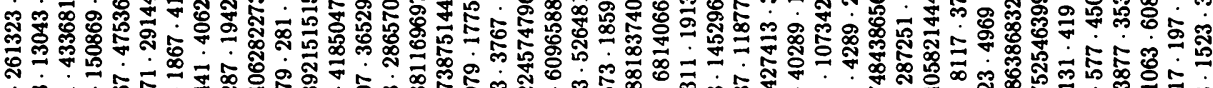

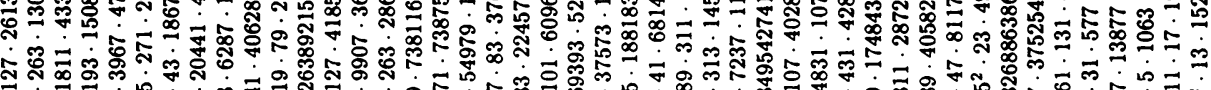

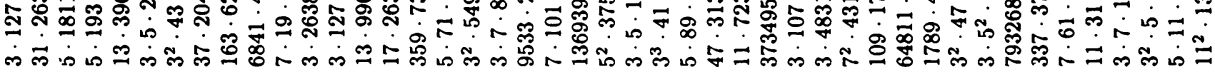

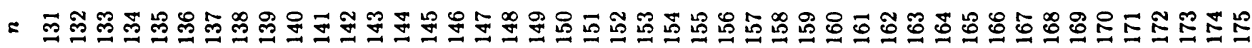

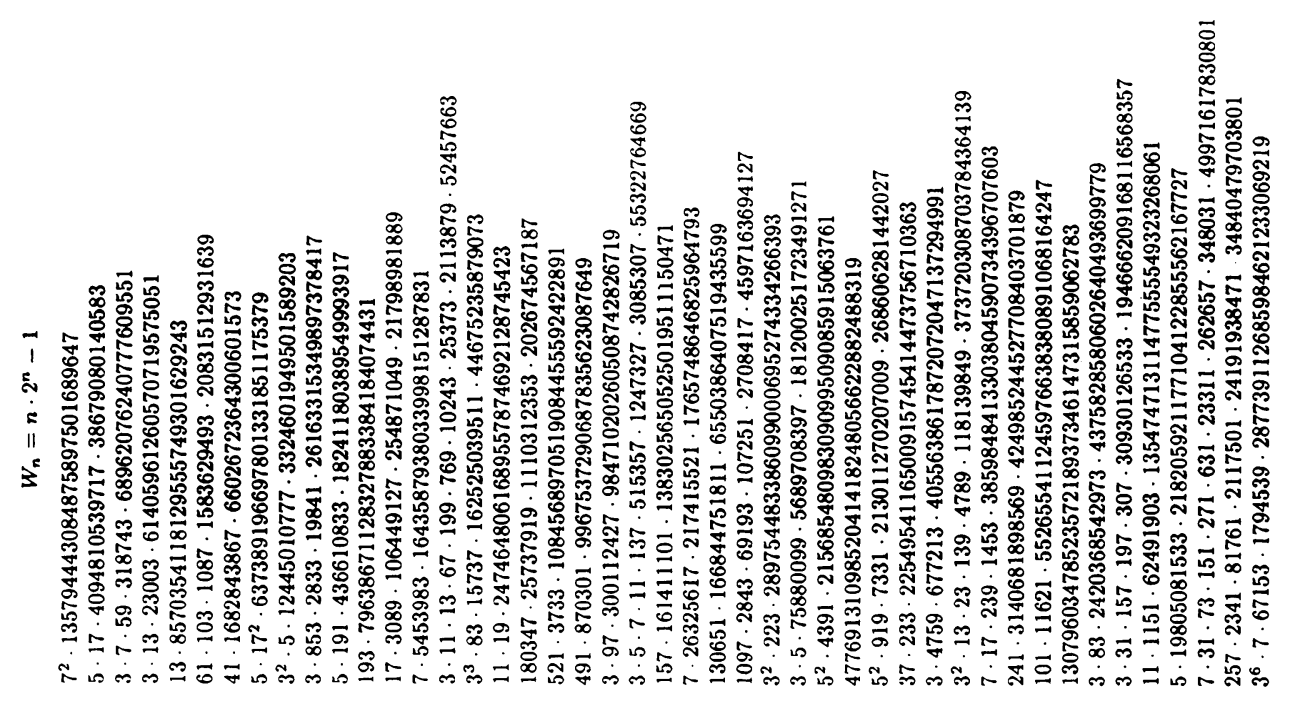

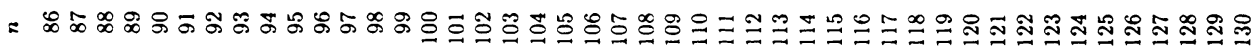



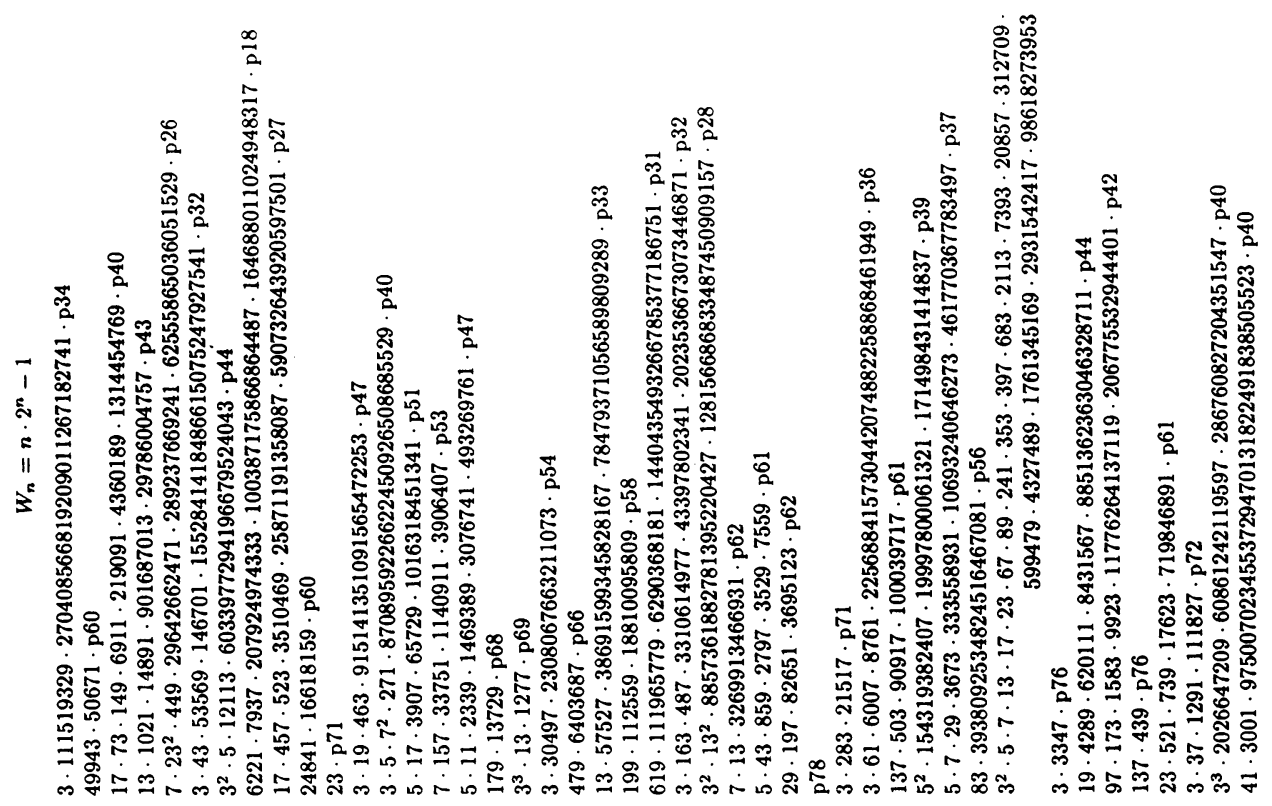

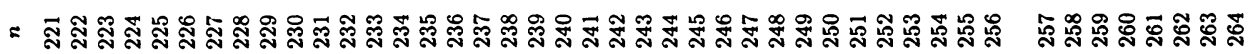

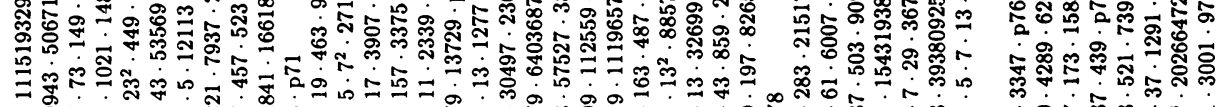

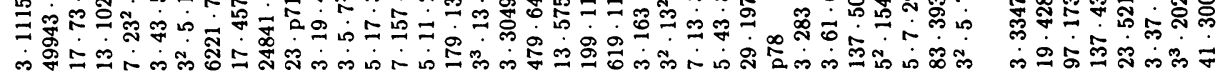

W

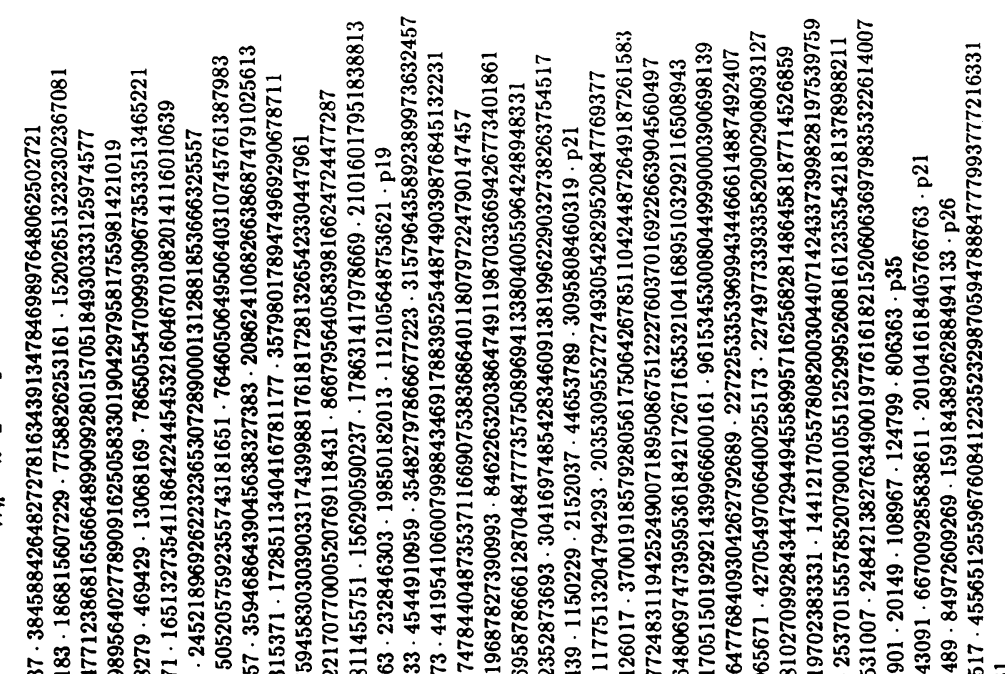

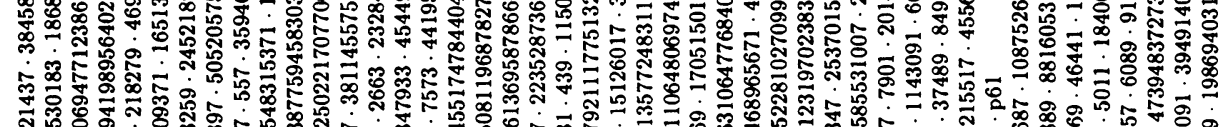

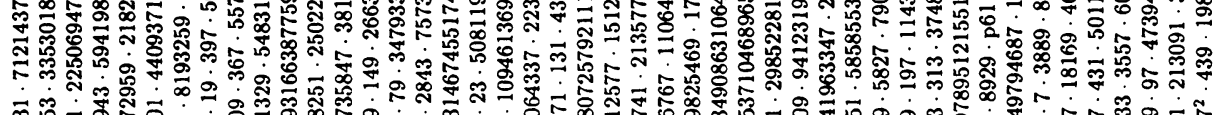

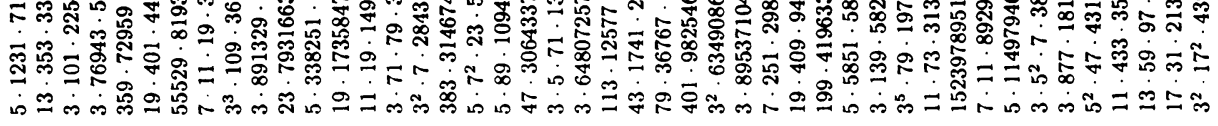

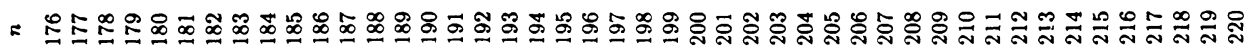




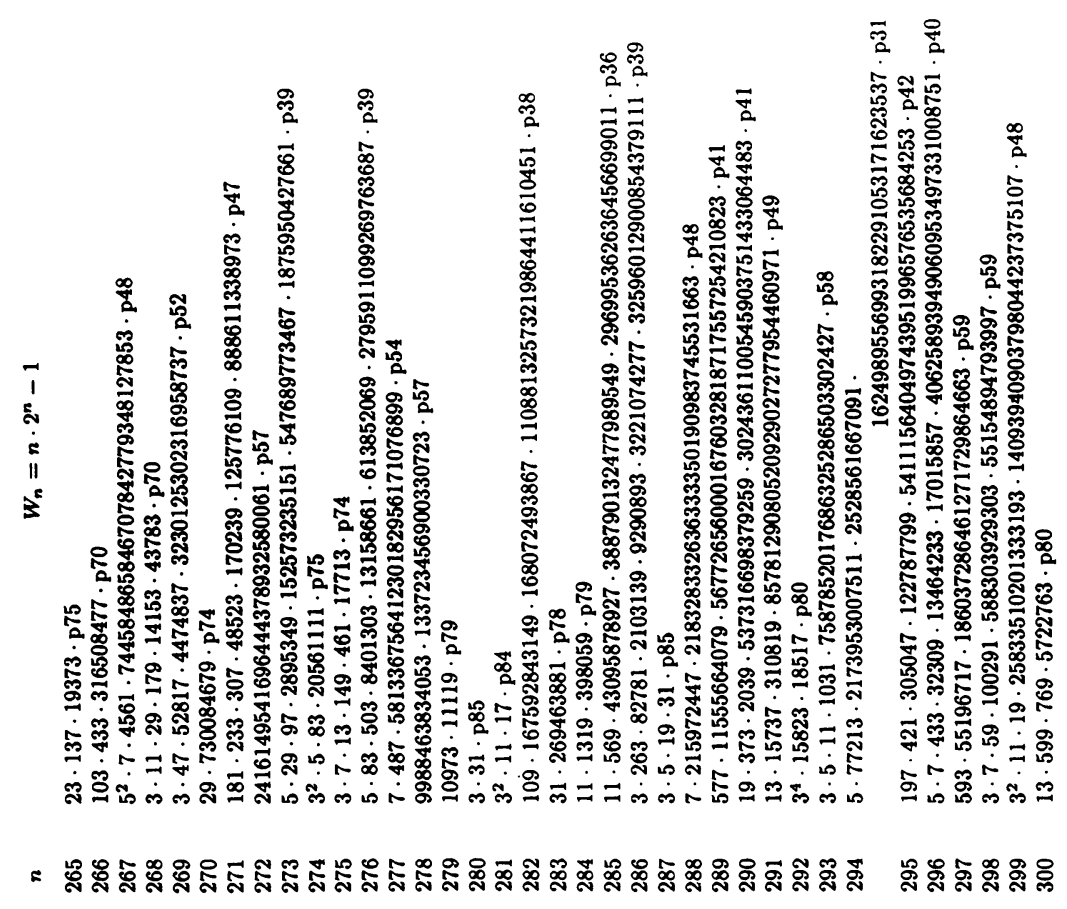

